\title{
IDENTIFICAÇÃO DA DUPLA EXCEPCIONALIDADE EM ADULTO: UM CASO DE ALTAS HABILIDADES/SUPERDOTAÇÃO E TDAH
}

\author{
Mariana Rodrigo do Vale Costa e Silva* \\ Rauni Jandé Roama-Alves** \\ Tatiana de Cássia Nakano*** \\ Bárbara David Rech ${ }^{* * * *}$
}

\begin{abstract}
RESUMO: O objetivo do presente artigo foi relatar um caso de avaliação neuropsicológica conduzida junto a um adulto que resultou no diagnóstico de dupla excepcionalidade relacionada à presença de Altas Habilidades/Superdotação (AH/SD) e Transtorno de Déficit de Atenção/Hiperatividade (TDAH). A avaliação contou com sete sessões compostas de administração de instrumentos psicológicos e neuropsicológicos, juntamente de observações clínico-qualitativas. Teve-se como aporte teórico para o atendimento o modelo de Competências funcionais em neuropsicologia clínica. Os dados foram agrupados em habilidades neurocognitivas avaliadas, a saber: inteligência, funções executivas chamadas frias/cognitivas, habilidades escolares e habilidades socioemocionais. De modo geral, os resultados demonstraram altos valores de Quociente Intelectual, mas déficits em funções executivas e em habilidades socioemocionais. O perfil apresentado pelo paciente foi discutido de acordo com a literatura na área, e, consequentemente, de modo que o identificou com dupla excepcionalidade entre AH/SD e TDAH. Discussões sobre a identificação tardia do quadro também são apresentadas, bem como sugestões para o suporte adequado às suas necessidades específicas.
\end{abstract}

PALAVRAS-CHAVE: Avaliação neuropsicológica, Diagnóstico, Identificação tardia.

\section{IDENTIFICATION OF TWICE EXCEPTIONALITY IN ADULTS: A CASE OF GIFTEDNESS AND ADHD}

\begin{abstract}
The aim of this article was to report a case of neuropsychological assessment conducted with an adult that resulted in the diagnosis of twice exceptionality related to the presence of Giftedness (AH/SD) and Attention deficit hyperactivity disorder (ADHD). The evaluation included seven sessions consisting of the administration of psychological and neuropsychological instruments, together with clinical-qualitative observations. The theoretical support for the service was the model of Functional competences in clinical neuropsychology. The data were grouped into assessed neurocognitive skills, namely: intelligence, executive functions called cold / cognitive, school skills and socioemotional skills. In general, the results showed high values of Intellectual Quotient, but deficits in executive functions and in socio-emotional skills. The profile presented by the patient was discussed according to the literature in the area, and, consequently, in a way that identified him with double exceptionality between AH/SD and ADHD. Discussions about late identification of the condition are also presented, as well as suggestions for the appropriate support for your specific needs.
\end{abstract}

KEYWORDS: Neuropsychological assessment, Diagnosis, Late identification.

\footnotetext{
* Mestrado em Psicologia pela Universidade Federal de Mato Grosso (UFMT). E-mail: marianarvcs@gmail.com. ORCID: https://orcid.org/0000-0003-2822-2724.

** Doutorado em Psicologia pela Pontifícia Universidade Católica de Campinas (PUC-Campinas). Docente na Universidade Federal de Rondonópolis (UFR). E-mail: rauniroama@gmail.com. ORCID: https://orcid.org/0000-0002-1982-1488.

*** Doutorado em Psicologia pela PUC-Campinas. Docente do Programa de Pós-Graduação em Psicologia da PUC-Campinas. E-mail: tatiananakano@hotmail.com. ORCID: https://orcid.org/0000-0002-5720-8940.

**** Mestranda em Psicologia pela UFMT. E-mail: barbararech.psi@gmail.com. ORCID: https://orcid.org/0000-0003-1544$\underline{5689}$.
}

\begin{tabular}{|l|l|l|l|l|l|l|}
\hline APRENDER - Cad. de Filosofia e Psic. da Educação & Vitória da Conquista & Ano XV & n. 26 & p. 26-42 & Jul./Dez. 2021 \\
\hline
\end{tabular}




\section{Introdução}

As Altas Habilidades/Superdotação (AH/SD) podem ser definidas como a presença de um potencial elevado em qualquer área, que proporciona destaque ao indivíduo. No Brasil, os superdotados são definidos como aqueles que apresentam um alto potencial, combinado ou isolado, nas áreas intelectual, acadêmica, de liderança e psicomotricidade, além de manifestar uma elevada criatividade, um alto envolvimento com a aprendizagem e também com a realização de tarefas de seu interesse (BRASIL, 2008).

Apesar desse potencial ser, mais comumente, identificado na infância, a literatura tem relatado casos em que somente foi confirmado na vida adulta. Tal situação pode ser compreendida, em partes, se considerarmos que o fenômeno no adulto tem se mostrado pouco estudado, sendo priorizadas as investigações na infância e adolescência (LANG et al., 2019). Consequentemente, pouco se sabe sobre o que acontece quando esse indivíduo se torna adulto (ALSHEHRI, 2020), sendo que tal lacuna tem dificultado a compreensão adequada das características, experiências e necessidades desse público específico.

De modo geral, se a identificação acontece na infância, provavelmente o potencial elevado nas áreas de criatividade, liderança ou capacidade acadêmica, por exemplo, se mantém à medida em que os indivíduos envelhecem (ALSHEHRI, 2020). Assim, podemos afirmar que todo adulto superdotado certamente foi uma criança superdotada, que apresentava certa facilidade em alguma área, notadamente nas tarefas escolares (ARANTES-BRERO, 2020). Já o inverso nem sempre é verdadeiro, visto que a ausência de suporte individualizado e atendimento educacional especializado, que atendam as necessidades particulares dos superdotados, pode acabar por trazer importantes prejuízos a esses indivíduos, de modo que sua habilidade elevada pode acabar se nivelando com seus pares de idade (SZYMANSKI; WRENN, 2019). Em outro oposto, quando adequadamente atendidos na sua infância, os adultos superdotados tendem a apresentar satisfação com a vida, tanto pessoal quanto profissional, sentido de vida definido, bem como maiores índices de bem-estar subjetivo (VOTTER; SCHNELL, 2019), apesar dos estudos dessa natureza ainda serem escassos (WIRTHWEIN; ROST, 2011).

Igualmente, achados contraditórios são relatados na literatura em relação ao funcionamento psicológico de adultos superdotados, ora enfatizando que o potencial elevado pode atuar como fator de proteção, ora como fator que pode ampliar suas vulnerabilidades (MATTA; GRITTI; LANG, 2019). Por tais motivos, a identificação se mostra importante, mesmo que realizada tardiamente. Isso porque, na vida adulta, os superdotados usualmente apresentam capacidades, potencialidades e recursos que atuam de modo a facilitar sua adaptação ao meio, embora um segundo grupo, não adaptado também seja encontrado (MOSQUERA; STOBAUS; FREITAS, 2014). 
O texto aqui apresentado irá se centrar na apresentação de um caso de avaliação neuropsicológica de um adulto com sinais indicadores de potencial elevado na área intelectual. A superdotação nessa área envolve a presença de altos níveis de potencial e desempenho em uma ou mais áreas que compõem a inteligência humana (STRICKER et al., 2020). Normalmente, a superdotação acadêmica demonstra um alto nível em habilidades convergentes, lógicas e hipotético-dedutivas (ZENASNI et al., 2016), bem como pontuações mais altas em capacidade mental geral e desempenho acadêmico (CASTEJÓN et al., 2016).

Ao mesmo tempo, sinais indicadores da presença de Transtorno de Déficit de Atenção e Hiperatividade (TDAH) também se faziam presentes no presente caso investigado, de modo que a hipótese da dupla excepcionalidade foi levantada. Tal quadro se refere a uma condição em que há combinação de AH/SD com algum déficit ou transtorno. Envolve, nesse sentido, o reconhecimento da possibilidade de que pessoas que demonstram capacidades superiores em uma ou mais áreas poderiam apresentar, ao mesmo tempo, deficiências ou condições incompatíveis com essas características (NAKANO; SIQUEIRA, 2012). De modo geral, tais indivíduos apresentam diferenças importantes em relação àqueles que apresentam somente superdotação ou somente deficiência (JOSEPHSON; WOLFGANG; MEHRENBERG, 2018).

Devido à complexidade do quadro, sua identificação ainda tem se mostrado um desafio e sendo marcada pela subnotificação. Isso porque, na prática, predomina a ausência de identificação correta devido a três possibilidades: (1) alunos que são identificados como superdotados mas sua incapacidade não é identificada, (2) estudantes identificados com déficit mas que não têm sua alta habilidade reconhecida, ou (3) tanto a AH/SD quanto a deficiência não são identificados (FOLEY-NICPON; KIM, 2018). Parte dessa situação pode ser compreendida perante a constatação de que, mais comumente, somente a deficiência é identificada (LEE; OLENCHAK,2018) ou tais indivíduos acabam sendo atendidos de acordo com o primeiro diagnóstico, seja ele de superdotação ou déficit (JOSEPHSON; WOLFGANG; MEHRENBERG, 2018).

A baixa conscientização acerca da dupla excepcionalidade e a crença de que estudantes com déficits e transtornos não podem ser superdotados (GENTRY; FUGATE, 2008), assim como a combinação de características que fogem do desenvolvimento típico e que, muitas vezes, se contradizem (VILARINHO-RESENDE; FLEITH; ALENCAR, 2016), acabam por originar um perfil irregular do indivíduo, com características atípicas, complexas e peculiares que dificultam sua identificação. Consequentemente, uma série de resultados negativos tem sido associada ao diagnóstico incorreto: isolamento social, baixa autoestima, depressão, ansiedade, desempenho insuficiente, baixo autoconceito, os quais podem afetar a saúde mental desses indivíduos (DARE; NOWICKI, 2015).

Nesse sentido, a literatura tem recomendado que sempre que houver uma suspeita de presença de um quadro de dupla excepcionalidade, o acesso a uma avaliação abrangente é essencial para determinar o nível de superdotação, o grau de comprometimento devido à deficiência, as áreas afetadas pela deficiência e o oferecimento de atendimento específico para as duas condições (GILMAN et al., 2013). 
Esta atuação conjunta deve buscar compreender o impacto da superdotação no transtorno e viceversa, bem como a identificação da etiologia das dificuldades que se fizerem presentes (GUIMARÃES; ALENCAR, 2012), dentro de um processo amplo e compreensivo de avaliação, voltado tanto à área de AH/SD quanto à deficiência (SILVERMAN, 2018).

Diante do exposto, o presente caso foi escolhido para ser relatado em vista da escassez de relatos clínicos acerca de dupla-excepcionalidade na vida adulta e de estudos sobre o psicodiagnóstico tardio nesta população. $\mathrm{O}$ objetivo do presente artigo foi relatar um caso de avaliação neuropsicológica conduzida junto a um adulto que resultou no diagnóstico de dupla excepcionalidade relacionada à presença de AH/SD e TDAH.

\section{Método}

Foi utilizado um delineamento de estudo de caso único (STAKE, 2006), de caráter transversal, com o objetivo de investigar as funções neuropsicológicas do paciente. Foram utilizadas sete sessões de avaliação, realizadas em clínica particular, cada uma com duração média de 50 minutos. A escolha da bateria de testes foi realizada de acordo com as demandas do próprio caso, a partir das análises quantitativas e qualitativas (READY; VEAGUE, 2014).

Dessa forma, teve-se como aporte teórico para o atendimento realizado o modelo de "Competências funcionais em neuropsicologia clínica", que inclui a eficácia na obtenção de um histórico abrangente; identificação dos principais problemas / questões neurocomportamentais a serem tratados; seleção apropriada e aplicação de uma ampla gama de procedimentos de avaliação neuropsicológica para diversas populações; aplicação de conhecimentos da teoria psicométrica (construção de testes, confiabilidade / validade, acurácia diagnóstica); estratégias interpretativas integrativas para diagnóstico diferencial; desenho e implementação de reabilitação e intervenções de apoio; e consultoria individual e de sistemas, aplicando a base de conhecimento atual em neurociências clínicas (REY-CASSERLY; ROPER; BAUER, 2012). Seguindo o modelo fornecido por France et al. (2008), divide-se as competências funcionais em competências baseadas no conhecimento e competências aplicadas, reconhecendo que todas devem ser tipicamente demonstradas de forma integrada pelo clínico. Os quatro domínios de competência são: prática baseada em evidências, avaliação, intervenção e consulta.

Além disso, ressalta-se que foram assegurados os processos éticos brasileiros para o presente relato de caso. De acordo com o Artigo $1^{\circ}$, parágrafo único da Resolução número 510, de 07 de abril de 2016 do Conselho Nacional de Saúde (BRASIL, 2016), é possibilitada a realização de "pesquisa que objetiva o aprofundamento teórico de situações que emergem espontânea e contingencialmente na prática profissional, desde que não revelem dados que possam identificar o sujeito". Sendo assim, garantidas a análise de todas essas variáveis e considerando o perfil dessa investigação, não foi necessário seu registro em algum Comitê de Ética de Pesquisa com Seres Humanos. 


\section{Participante}

O caso aqui relatado foi de "B.”, 51 anos, avaliado entre os meses de janeiro e março de 2021, na cidade de Cuiabá, Mato Grosso, Basil. Tal participante buscou avaliação após seu filho iniciar o mesmo processo em razão de queixas de dificuldades de aprendizagem. B. é funcionário público concursado, possui duas graduações e três pós-graduações, é casado e tem dois filhos, sendo um adolescente e o outro criança. A queixa principal apresentada por ele foi em especial à disciplina matemática, além de desatenção e dificuldades para processar informações de modo rápido.

\section{Procedimentos}

A avaliação contou com sete sessões compostas de administração de instrumentos psicológicos e neuropsicológicos, juntamente de observações clínico-qualitativas. Após cada atendimento, era feito o relato em prontuário com uma descrição da sessão, bem como as impressões e observações da psicóloga a respeito do comportamento e relatos do paciente. Esse prontuário era de uso exclusivo da psicóloga, no qual também realizava a guarda das folhas de respostas dos testes. Ao final da avaliação, os dados foram agrupados em habilidades neuropsicológicas avaliadas, a saber: inteligência, funções executivas chamadas "frias"/cognitivas, habilidades escolares e habilidades socioemocionais. O laudo foi produzido e realizada devolutiva ao paciente. Todo documento produzido (prontuário, respostas aos testes, laudos, entre outros) foram arquivados por no mínimo cinco anos. Na sequência, para publicação, esses dados foram confrontados com a literatura das áreas da neuropsicologia e das altas habilidades/superdotação.

\section{Instrumentos e análise de dados}

A literatura tem recomendado a inclusão de diferentes métodos na avaliação das AH/SD, que considerem aptidões gerais e cognitivas, habilidades específicas e traços não cognitivos (ZAIA; NAKANO, 2020), visando, dependendo do caso, a avaliação de aspectos como pensamento divergente, nível intelectual, autoconceito, aptidões e criatividade, podendo ser complementados por meio do uso de questionários de interesses, escalas de ajustamento social e emocional, entrevistas e instrumentos para avaliação da personalidade (METTRAU; REIS, 2007).

Nesse sentido, os instrumentos selecionados para a presente avaliação foram: a) roteiro de entrevista de anamnese semiestruturada; b) Escala de Inteligência Wechsler para Adultos - Terceira Edição (WAIS-III) (WECHSLER, 2004); c) Escala de Avaliação de Disfunções Executivas de Barkley (BDEFS) (BARKLEY, 2018); d) Teste de Aprendizagem Auditivo-Verbal (RAVLT) (REY, 2018); e) Figuras Complexas de Rey (REY, 2014); f) Bateria Psicológica para Avaliação da Atenção (BPA) (RUEDA, 2013); g) Tarefa de Leitura de Palavras e Pseudopalavras (TLPP) (RODRIGUES; MINÁ; SALLES, 2017a); h) Tarefa de Escrita de Palavras e Pseudopalavras (TEPP) (RODRIGUES; MINÁ; 
SALLES, 2017b); e i) Escala de Transtorno de Déficit de Atenção e Hiperatividade - Versão Adolescentes e Adultos (ETDAH-AD) (BENCZIK, 2013). A descrição de cada instrumento utilizado pode ser conferida no Quadro 1.

Quadro 1 - Apresentação dos instrumentos psicométricos utilizados para a avaliação neuropsicológica de B.

\begin{tabular}{|c|c|}
\hline Instrumento & Objetivo \\
\hline $\begin{array}{c}\text { Escala de Inteligência Wechsler para } \\
\text { Adultos - Terceira Edição (WAIS-III) }\end{array}$ & $\begin{array}{c}\text { Medir o coeficiente de inteligência (Q.I.) de uma pessoa, tanto o total como } \\
\text { especificado em seus subdomínios (total de 14 subtestes). }\end{array}$ \\
\hline $\begin{array}{c}\text { Escala de Avaliação de Disfunções } \\
\text { Executivas de Barkley (BDEFS) }\end{array}$ & $\begin{array}{c}\text { Avaliar as disfunções executivas do indivíduo baseado em cinco domínios: } \\
\text { gerenciamento de tempo, organização/resolução de problemas, } \\
\text { autocontrole, motivação e regulação emocional. }\end{array}$ \\
\hline $\begin{array}{c}\text { Teste de Aprendizagem Auditivo-Verbal } \\
\text { (RAVLT) }\end{array}$ & $\begin{array}{c}\text { Avaliar a memória verbal episódica e fornecer informações sobre as } \\
\text { medidas de aprendizagem auditivo-verbal, índices de interferência e de } \\
\text { retenção de informações, e memória de reconhecimento. }\end{array}$ \\
\hline Figuras Complexas de Rey & Avaliar a atividade perceptiva e a memória visual \\
\hline $\begin{array}{c}\text { Bateria Psicológica para Avaliação da } \\
\text { Atenção (BPA) }\end{array}$ & $\begin{array}{c}\text { Averiguar possíveis problemas relacionados a atenção concentrada, dividida } \\
\text { e alternada }\end{array}$ \\
\hline $\begin{array}{c}\text { Tarefa de Leitura de Palavras } \\
\text { e Pseudopalavras (TLPP) }\end{array}$ & $\begin{array}{c}\text { Identificar a integridade das rotas de leitura (fonológica, lexical ou ambas) } \\
\text { para complementar o processo de avaliação e diagnóstico de possíveis } \\
\text { dificuldades e prejuízos em habilidades acadêmicas }\end{array}$ \\
\hline $\begin{array}{c}\text { Tarefa de Escrita de Palavras } \\
\text { e Pseudopalavras (TEPP) }\end{array}$ & $\begin{array}{c}\text { Identificar a integridade das rotas de escrita (fonológica, lexical ou ambas) } \\
\text { para complementar o processo de avaliação e diagnóstico de possíveis } \\
\text { dificuldades e prejuízos em habilidades acadêmicas }\end{array}$ \\
\hline $\begin{array}{c}\text { Escala de Transtorno do Déficit de } \\
\text { Atenção e Hiperatividade - Versão } \\
\text { Adolescentes e Adultos (ETDAH-AD) }\end{array}$ & $\begin{array}{c}\text { Auxiliar no processo diagnóstico do TDAH, com a possibilidade de } \\
\text { distinguir a apresentação do transtorno, a intensidade e o nível de prejuízo } \\
\text { existente }\end{array}$ \\
\hline
\end{tabular}

Fonte: Produção dos autores

Tais instrumentos foram selecionados para compor os procedimentos de avaliação por serem considerados instrumentos válidos e favoráveis pelo Sistema de Avaliação de Testes Psicológicos SATEPSI, e que buscam avaliar diversas habilidades cognitivas que poderiam estar associadas aos prejuízos relatados na entrevista de anamnese. Desta forma, foi possível verificar se as hipóteses diagnósticas estariam corretas e também excluir a presença de outras condições comórbidas ou conflitantes.

\section{Resultados}

Optou-se por apresentar os resultados de acordo com as habilidades neuropsicológicas que foram avaliadas. Primeiramente, são apresentadas as habilidades relacionadas com inteligência, que se refere à capacidade de o indivíduo pensar de forma abstrata, resolver problemas e aprender com a experiência (MECCA; SÁ; MACEDO, 2018). Posteriormente, são apresentadas as habilidades relacionadas às funções executivas chamadas "frias"/cognitivas, que são os processos cognitivos que atuam de forma integrada e que permitem direcionar processos mentais e comportamentos a objetivos com enfoque em aspectos mais lógicos e abstratos (COSTA et al., 2020). São também apresentados os resultados relacionados às habilidades escolares, que são um conjunto de conhecimentos organizados e aprendidos mais contundentemente em âmbito escolar (VIAPIANA et al., 2016). Por fim, relatam-se as 
habilidades socioemocionais averiguadas, que envolvem comportamentos e emoções, e suas adaptações em âmbitos intra e interpessoais (TAYLOR et al., 2017).

\section{Inteligência}

As habilidades avaliadas acerca da inteligência de B. foram inteligência fluida (visuoespacial), inteligência cristalizada (verbal), velocidade de processamento visual e verbal, atenção visual e verbal, memória visual de curto e longo prazo, e memória visual de curto e longo prazo. O Quadro 2 apresenta, de forma sintetizada, o desempenho de B. em cada habilidade e o respectivo teste utilizado para sua avaliação.

Quadro 2 - Resumo do desempenho de B. em habilidades relacionadas à inteligência

\begin{tabular}{|c|c|c|}
\hline Habilidades & Desempenho & Teste de Referência \\
\hline Inteligência fluida (visuoespacial) & Acima da média & $\begin{array}{l}\text { WAIS-III (Q.I. de Execução; subteste } \\
\text { Raciocínio Matricial) }\end{array}$ \\
\hline Inteligência cristalizada (verbal) & Acima da média & WAIS-III (Q.I. Verbal; ICV) \\
\hline Velocidade de processamento visual & Acima da média & WAIS-III (IVP) \\
\hline Atenção verbal & Abaixo da média & BPA; ETDAH-AD \\
\hline Atenção visual & Abaixo da média & BPA; ETDAH-AD \\
\hline Memória de curto prazo verbal & Abaixo da média & RAVLT \\
\hline Memória de longo prazo verbal & Dentro da média & RAVLT \\
\hline Memória de curto prazo visual & Dentro da média & $\begin{array}{c}\text { WAIS-III (subteste Completar Figuras; Figuras } \\
\text { Complexas de Rey) }\end{array}$ \\
\hline
\end{tabular}

Fonte: Produção dos autores Complexas de Rey

Legenda: Q.I. = Quociente de Inteligência; ICV = Índice de Compreensão Verbal; IVP = Índice de Velocidade de Processamento.

Dentre os resultados sobre essa habilidade que mais chamaram a atenção, destacam-se algumas. Pode-se relatar a princípio, os resultados dos testes de avaliação de inteligência fluida e cristalizada que apontaram desempenhos considerados Superior (entre 121 e 130) e Muito Superior (> 130). Dentre seus testes de referência, observa-se que no coeficiente de inteligência (QI) de execução, a pontuação foi 144, o QI verbal, 142, e o Índice de Compreensão Verbal, 127. O escore ponderado do subteste Raciocínio Matricial foi de 15, em uma variação de 1 a 19, na qual quanto maior, melhor o desempenho, com 10 sendo a média. Sendo assim, B. apresentou uma pontuação superior também em tal subteste. Da mesma forma, a velocidade de processamento foi considerada Superior pela pontuação no Índice de Velocidade de Processamento de 124.

O desempenho de B. foi abaixo da média nas seguintes habilidades: atenção e memória de curto prazo verbal. Essas habilidades foram analisadas a partir dos escores da BPA, que foram 20 (inferior) para Atenção Total, e 30 (médio inferior), 40 (médio inferior) e 10 (inferior) para Atenção Concentrada, Atenção Dividida e Atenção Alternada, respectivamente. Convergindo com esses escores, a subescala Desatenção da ETDAH-AD somou 67 pontos, equivalente ao percentil 95, com classificação 
Superior, indicando alta desatenção. Por fim, o resultado que convergiu com o desempenho prejudicado em memória de curto prazo verbal se deu pela RAVLT, cujo Escore Total e o índice de Aprendizagem ao Longo das Tentativas foram enquadrados no percentil 25, com classificação Médio Inferior.

\section{Funções Executivas "Frias"/ Cognitivas}

As funções executivas chamadas "frias" ou cognitivas avaliadas em B. foram planejamento, flexibilidade cognitiva, controle inibitório, memória de trabalho visual e verbal, e integração visuomotora. O Quadro 3 apresenta um resumo do desempenho do paciente em cada habilidade e o teste de referência utilizado para fazer a avaliação.

Quadro 3 - Resumo do desempenho de B. em habilidades relacionadas à funções executivas "frias"

\begin{tabular}{|c|c|c|}
\hline Habilidades & Desempenho & Teste de Referência \\
\hline Planejamento & Abaixo da média & $\begin{array}{c}\text { BDEFS (Gerenciamento de Tempo e } \\
\text { Organização/Resolução de Problemas) }\end{array}$ \\
\hline Flexibilidade cognitiva & Dentro da média & WAIS-III (subtestes Semelhanças e Códigos); BPA \\
\hline Controle inibitório & Abaixo da média & ETDAH-AD; BDEFS (Autocontrole) \\
\hline Memória de Trabalho Visual & Dentro da média & WAIS-III (subtestes Arranjo de Figuras e Cubos) \\
\hline Memória de Trabalho Verbal & Acima da média & WAIS-III (IMO) \\
\hline Integração visuomotora & Dentro da média & WAIS-III (IOP); Figuras Complexas de Rey \\
\hline
\end{tabular}

Fonte: Produção dos autores

Legenda: IMO = Índice de Memória Operacional; IOP - Índice de Organização Perceptual.

Entre as habilidades relacionadas às funções executivas, destaca-se o desempenho acima da média para memória de trabalho verbal, aferido por meio do Índice de Memória Operacional da WAISIII, que teve pontuação de 125, cuja classificação é Superior. Contudo, cabe ressaltar também os desempenhos abaixo da média em planejamento e controle inibitório. O planejamento foi avaliado a partir das pontuações das subescalas Gerenciamento de Tempo e Organização/Resolução de Problemas da BDEFS, correspondentes respectivamente aos percentis 95 (Levemente limítrofe) e 90 (Limítrofe) quando comparados à amostra por escolaridade. Por último, o controle inibitório, foi avaliado por meio da subescala Autocontrole da BDEFS, cuja pontuação, 38, corresponde ao percentil 85, classificado como limítrofe. Conjuntamente, a pontuação da subescala de Impulsividade da ETDAH$\mathrm{AD}$ foi de 48, correspondente ao percentil 70, que indica classificação Médio Superior, e é correspondente a altos índices de impulsividade.

\section{Habilidades escolares}

As habilidades escolares foram avaliadas em B. em razão da suspeita de Transtorno Específico de Aprendizagem, pois uma de suas queixas mais frequentes era de dificuldades de aprendizagem. Avaliouse a linguagem escrita, especificamente leitura e escrita, e o raciocínio matemático. O Quadro 4 mostra o desempenho apresentado pelo paciente nessas habilidades e os testes utilizados para sua avaliação. 
Quadro 4 - Resumo do desempenho de B. em habilidades escolares

\begin{tabular}{|c|c|c|}
\hline Habilidades & Desempenho & Teste de Referência \\
\hline Linguagem escrita (leitura) & Dentro da média & TEPP \\
\hline Linguagem escrita (escrita) & Dentro da média & WAIS-III (subteste Aritmética) \\
\hline Raciocínio matemático & Acima da média & \\
\hline
\end{tabular}

Fonte: Produção dos autores

Os resultados correspondentes às habilidades de linguagem escrita, tanto de leitura como de escrita, foram avaliados por meio dos percentis por palavras e pseudopalavras da TLPP e TEPP, respectivamente. Para ambos, o desempenho foi considerado dentro da média, com percentis acima de 25, de forma que as pontuações obtidas pelo paciente, a saber, foi 90 tanto para palavras como pseudopalavras na TLPP, e 30 para palavras com 50 para pseudopalavras na TEPP. Ou seja, o paciente apresentou desempenhos compatíveis ao esperado para seu nível acadêmico, sem dificuldades. Além disso, cabe ressaltar que, qualitativamente, também não apresentou dificuldades em escrita em nenhuma outra atividade que a requeria, inclusive, em que a exigia de forma mais complexa. Também não foram identificadas dificuldades em relação à compreensão de textos, com leitura em velocidade adequada. Por sua vez, em raciocínio matemático, avaliado pelo subteste Aritmética da WAIS-III, obteve um escore ponderado de $14, \quad$ e, portanto, considerado acima da média. Inclusive, realizou tal atividade, clinicamente, com facilidade.

\section{Habilidades socioemocionais}

Qualitativamente, algumas características comportamentais e emocionais precisam ser relatadas sobre o caso de B. Durante as sessões, se mostrou como uma pessoa comunicativa, erudita, que gostava de explicar e saber a explicação das coisas, característica essa muito intensa. Apesar de relatar traumas e dificuldades no âmbito escolar, graduou-se em três cursos superiores, entre os cinco que iniciou ao longo da vida. Se mostrava muito metódico na realização das atividades, como não conseguir passar para um próximo item caso tivesse tido dificuldades no anterior. Entretanto, não revisava suas respostas ao encerrar determinada tarefa, sendo impulsivo, o que favoreciam erros. Minucioso, se mostrava muito afeito a detalhes e gostava de mostrar que os havia percebido. Apesar de comprometido com o processo, o paciente raramente chegava no horário para as sessões, com atrasos recorrentes de 10 até 30 minutos. Das sete sessões, apenas uma iniciou-se no horário marcado. Adicionalmente, B. relatou diversas dificuldades relacionadas ao planejamento espacial, como o costume de esbarrar e bater em móveis com frequência e se perder em algumas rotas conhecidas quando não prestava atenção. Diante das dificuldades percebidas, o paciente relatou também se sentir ansioso e inseguro. Em determinados momentos, relatou evitar falar ou responder a determinadas perguntas mesmo tendo certeza da resposta. Contou se sentir envergonhado também quando erra a escrita de determinadas palavras por seu pensamento fluir mais rapidamente do que seus movimentos motores. 


\section{Discussão}

O objetivo do presente artigo foi relatar um caso de avaliação neuropsicológica conduzida junto a um adulto que resultou no diagnóstico de dupla excepcionalidade relacionada à presença de AH/SD e TDAH. A literatura da área aponta que, ainda que sejam condições diferenciadas, o TDAH e as AH/SD apresentam características que podem ser similares, como: impulsividade, fala acelerada, aumentada sensibilidade a estimulação ambiental, intensa curiosidade, constantes queixas comportamentais provindas dos cuidadores e dificuldades de adaptação a ambientes novos (KAUFMANN et al., 2000; OUROFINO; FLEITH, 2005; ALVES; NAKANO, 2015; OGEDA; CHACON, 2020). Ademais, também são descritas a ocorrência de características específicas para indivíduos que apresentam dupla excepcionalidade entre o TDAH e as $\mathrm{AH} / \mathrm{SD}$, como, desempenho escolar inconstante, déficit em habilidades motoras finas, interesses não acadêmicos e maior potencialidade para obtenção de novos conhecimentos (ALVES, NAKANO, 2015; ANTSHELL et al., 2008, ZENTALL et al., 2011). Torna-se interessante notar que B. apresenta características de ambas excepcionalidades, diferenciando-se de indivíduos com apenas AH/SD ou TDAH (JOSEPHSON; WOLFGANG; MEHRENBERG, 2018).

No presente caso, foi verificado que B. apresentou Q.I Total de 146. No contexto das AH/SD, o critério mais comumente utilizado para identificar um potencial intelectual elevado envolve um desempenho que corresponde a dois desvios padrões acima da média, ou seja, Q.I. de 130 (LEE; OLENCHAK, 2018). Considerando-se o resultado apresentado por B., a superdotação nessa área foi confirmada. Tal tipo de AH/SD é representada por desempenho elevado relacionado a habilidades convergentes, lógicas e hipotético-dedutivas (ZENASNI et al., 2016), bem como pontuações mais altas em capacidade mental geral e desempenho acadêmico (CASTEJÓN et al., 2016), exatamente as áreas do WAIS-III em que o desempenho se mostrou acima da média (velocidade de processamento, inteligência fluida e cristalizada).

Mesmo B. apresentando inteligência superior, apresentou déficits de funções executivas frias, podendo ser essa última característica compatível com um diagnóstico de TDAH, de acordo com a literatura em neuropsicologia na área (CASTRO, 2018; MAGNIN; MAURS, 2017; MULLET; RINN, 2015; MICHELS, 2014). De acordo com o modelo proposto por Dyamond (2013), as funções executivas podem ser divididas em: memória de trabalho, controle inibitório e flexibilidade cognitiva.

Nesses aspectos, B. apresentou desempenho rebaixado em planejamento e controle inibitório, embora tenha alcançado desempenho dentro e acima da média em tarefas de memória de trabalho visual e verbal. As dificuldades referentes aos déficits de funções executivas possuem grande impacto na qualidade de vida no indivíduo (MULLET; RINN, 2015; CASTRO; LIMA, 2018; MAGNIN; MAURS, 2017; MICHELS, 2014). Em âmbito comportamental, e ao serem analisados os critérios diagnósticos 
para TDAH (APA, 2014), B. se enquadrou na maioria deles, inclusive, não fechou critérios para outra condição neuropsiquiátrica de acordo com o DSM-5 (APA, 2014).

O paciente apresentou características comportamentais, de dificuldades de planejamento de tempo e espacial, dificuldades em atenção, impulsividade. Relatou também se sentir ansioso e inseguro. Todo esse perfil foi relatado como presente desde a infância. Tais características foram compatíveis com os critérios diagnósticos para TDAH (APA, 2014), tais como: a) um padrão persistente de desatenção e/ou hiperatividade-impulsividade que interfere no funcionamento e no desenvolvimento; b) sintomas de desatenção ou hiperatividade-impulsividade estavam presentes antes dos 12 anos de idade; c) sintomas de desatenção ou hiperatividade-impulsividade estão presentes em dois ou mais ambientes; d) há evidências claras de que os sintomas interferem no funcionamento social, acadêmico ou profissional ou de que reduzem sua qualidade; e) os sintomas não ocorrem exclusivamente durante o curso de esquizofrenia ou outro transtorno psicótico e não são mais bem explicados por outro transtorno mental.

A complexidade do caso de B. pode ter contribuído para sua subnotificação ao decorrer dos anos (FOLEY-NICPON; KIM, 2018; VILARINHO-RESENDE; FLEITH; ALENCAR, 2016), de modo que tanto as AH/SD quanto o TDAH não foram identificados, nem de forma isolada. É importante explicitar que o diagnóstico, em si, não diminuirá os déficits e queixas diárias apontadas por B. Ainda assim, como apontado por McCoach, Sieglen e Rubenstein (2020), o diagnóstico errôneo pode trazer consequências adversas para o indivíduo, como, não ter acesso as orientações, direitos e terapêuticas adequadas. Diversos prejuízos acadêmicos, pessoais e profissionais podem ter decorrido da falta dessa identificação na infância, especialmente se considerarmos que o conhecimento, por parte do indivíduo, de que as diversas dificuldades e problemas que ele apresenta são oriundos de um transtorno e não por incompetência ou falha consigo mesmo, pode incidir no aumento da qualidade de vida e autoestima, como demonstrado por Michels e Gonçalves (2014).

As habilidades escolares de B., de acordo com o resultado da avaliação, encontram-se dentro da média, o que foi incompatível com a queixa. Tal resultado pode ser compreendido perante a confirmação da dupla excepcionalidade aventada entre TDAH e AH/SD. Neste quadro, a diferença entre a capacidade ou potencial do indivíduo e o que ele efetivamente realiza podem se mostrar inconsistentemente discrepantes (TENTES et al., 2014). Diante desse resultado, a hipótese elaborada para sua aplicação se ancora na possibilidade de que o potencial elevado tenha atuado como fator de proteção e/ou mascarou o processo de aprendizagem de B. (ARIZAGA et al., 2016; JOSEPHSON; WOLFGANG; MEHRENBERG， 2018; MAYES; MOORE， 2016). Em alguns momentos de sua vida apresentou facilidades para aprender, por exemplo, finalizou três graduações e obteve bom desempenho nos testes da presente avaliação, e em outros não, como na infância, na qual apresentou dificuldades de aprendizagem. É sabido que na maioria dos casos de TDAH isoladamente há tais dificuldades, mesmo quando não comórbidos com Transtornos Específicos de Aprendizagem (APA, 2014). 
Referente às questões emocionais apresentadas por B., descritas como episódios de ansiedade, impulsividade, insegurança e vergonha pelos erros cometidos, a literatura sobre dupla excepcionalidade tem ressaltado, de forma comum, o enfrentamento de sentimentos relacionados à solidão e baixo autoconceito (MAYES; MOORE, 2016), ansiedade, frustração, dificuldades sociais e emocionais e diminuição da sensação de bem-estar (WORMALD; BANNISTER-TYRRELL, 2019), dificuldades de autoaceitação, autoconceito frágil, sentimentos de desconforto social, desinteresse escolar, perfeccionismo e vergonha (LUPART; TOY, 2009). Especialmente a associação entre AH/SD e TDAH, pode-se marcar pela presença de alta sensibilidade a críticas, desorganização, baixa autoestima, baixo autoconceito, intensidade emocional, dificuldade de desenvolver relacionamentos próximos, isolamento social, dificuldade em regular suas emoções (ANTSHEL; HENDRICKS; CONEJEROSSOLAR., 2018; GENTRY; FUGATE, 2018; LEE; OLENCHAK, 2018; KAUFMAN; KALBFLEISCH; CASTELLANOS, 2000). É comum que sem a identificação indivíduos com dupla excepcionalidade se sintam deslocados e incompreendidos (SILVERMAN, 2018), sendo as intervenções essenciais para a melhoria do bem-estar e ajustamento emocional desses indivíduos.

\section{Considerações Finais}

O presente estudo permitiu o relato de um processo de avaliação neuropsicológica que resultou em um diagnóstico de dupla excepcionalidade, composto pela associação entre TDAH e AH/SD, dentro de um processo tardio de identificação, o qual ocorreu somente na vida adulta, apesar dos indicadores relatados pelo paciente durante a infância. Essa situação reforça a percepção da literatura de que a condição da dupla excepcionalidade pode nunca ser identificada, sendo possível pensar que a integração entre essas condições possa ter dificultado ambos os diagnósticos. No caso de B., nem a condição do TDAH e nem das AH/SD foram identificadas anteriormente.

A ausência de identificação, no entanto, deu origem a uma série de indicadores relacionados às vulnerabilidades emocionais, mesmo ele tendo, inclusive, conseguido se incluir socialmente com, por exemplo, finalizar três graduações, ter sido aprovado em um concurso público e ter construído uma rede familiar de apoio. As questões emocionais decorrentes do quadro, relatadas pelo próprio paciente e observadas ao longo do processo avaliativo merecem ser alvo de atenção dado os impactos negativos que podem provocar no bem-estar e qualidade de vida de B. Um trabalho psicoterápico poderá beneficiá-lo dentro de um processo voltado ao autoconhecimento, regulação emocional e desenvolvimento de um autoconceito positivo, como foi aconselhado no laudo.

Espera-se que esse relato de caso possa conscientizar os profissionais que atuam na avaliação neuropsicológica, acerca da existência da possibilidade de ocorrência, conjunta, de transtornos e deficiências com a superdotação. Tal conhecimento se mostra essencial para que um diagnóstico correto desses indivíduos possa ser feito, conhecendo-se os efeitos da interação entre os dois quadros. É preciso 
que o paradigma de uma avaliação que identifique potenciais para além da identificação de déficits seja estabelecido nas práticas profissionais do neuropsicólogo de forma urgente.

\section{REFERÊNCIAS}

ALSHEHRI, Ranna Mohammad. Gifted adults career as a reflection of gifted education: a systematic review of the literature. IOSR International Journal of Research \& Method in Education, v. 10, n. 2, p. 38-45, 2020. Disponível em: https://www.iosrjournals.org/iosr-jrme/papers/Vol-10\%20Issue-2/Series6/G1002063845.pdf. Acesso em: 20 ago. 2021.

ALVES, Rauni Jandé Roama; NAKANO, Tatiana de Cássia. Criatividade em indivíduos com transtornos e dificuldades de aprendizagem: revisão de pesquisas. Psicologia Escolar e Educacional, Maringá, v. 19, n. 1, p. 87-96, 2015. Disponível em:

https://www.scielo.br/j/pee/a/xLg6VY3FW6DZtDgHWKMknPS/abstract/?lang=pt. Acesso em: 20 ago 2021.

AMERICAN PSYCHIATRIC ASSOCIATION (APA). DSM-5: Manual diagnóstico e estatístico de transtornos mentais. Porto Alegre, Artmed Editora, 2014.

ANTSHEL, Kevin M.; HENDRICKS, Kaitlin; FARAONE, Stephen V.; GORDON, Michael. Disorder versus disability: the challenge of ADHD in the context of a high IQ. The ADHD Report, v. 19, n.11, p. 4-8, 2011. Disponível em:

https://www.researchgate.net/publication/274291138 Disorder Versus Disability The Challenge of ADHD in the Context of a High IQ. Acesso em: 20 ago. 2021.

ARANTES-BRERO, Denise Rocha Belfort. Altas habilidades/superdotação na vida adulta: modos de ser e trajetórias de vida. Curitiba: Juruá, 2020.

ARIZAGA, Maria Paz Gómez; CONEJEROS-SOLAR, Maria Leonor; RODRÍGUEZ, Katia Sandoval.; SOLÍS, Solange Armijo. Doble excepcionalidad: análisis exploratorio de experiencias y autoimagen en estudiantes chilenos. Revista de Psicología, v.34, n.1, p. 5-37, 2016. Disponível em: http:/ / www.scielo.org.pe/scielo.php? script $=$ sci arttext\&pid=S0254-92472016000100002. Acesso em: 20 ago. 2021.

BALDWIN, Lois; OMDAL, Stuart; PERELES, Daphne. Beyond stereotypes: Understanding, recognizing, and working with twice-exceptional learners. Teaching Exceptional Children, v. 47, n. 4, p. 216-225, 2015. Disponível em: https://journals.sagepub.com/doi/10.1177/0040059915569361. Acesso em: 20 ago. 2021.

BARKLEY, Russell. Escala de Avaliação de Disfunções Executivas de Barkley - BDEFS. Manual. Trad. Godoy, P. Mattos e Leandro Malloy-Diniz. São Paulo, Hogrefe, 2018.

BENCZIK, Edyleine Bellini Peroni. Escala de Transtorno do Déficit de Atenção e Hiperatividade (ETDAH$A D)$ : versão adolescentes e adultos. São Paulo, Vetor, 2013.

BRASIL. Ministério da Educação. Política Nacional de Educação Especial na Perspectiva da Educação Inclusiva, Brasília. DF, 07 jan. 2008. Disponível em:

http://portal.mec.gov.br/arquivos/pdf/politicaeducespecial.pdf. Acesso em: 05 maio 2019.

BRASIL. Resolução n 510, de 07 de abril de 2016. Conselho Nacional de Saúde, 2016. Disponível em http://conselho.saude.gov.br/resolucoes/2016/Reso510.pdf. Acesso em: 02 ago. 2020. 
CASTEJÓN, Juan Luis et al. Latent class cluster analysis in exploring different profiles of gifted and talented students. Learning and Individual Differences, v. 50, p. 166-174, 2016. Disponível em: https://psycnet.apa.org/record/2016-46958-019. Acesso em: 20 ago. 2021.

CASTRO, Carolina Xavier Lima; LIMA, Ricardo Franco. Consequências do transtorno do déficit de atenção e hiperatividade (TDAH) na idade adulta. Revista Psicopedagia, v. 35, n.106, p. 61-72, 2018. Disponível em: http://pepsic.bvsalud.org/scielo.php?script=sci arttext\&pid=S010384862018000100008. Acesso em: 21 ago. 2021.

CONEJEROS-SOLAR, Maria Leonor et al. Aportes a la comprensión de la doble excepcionalidad: Alta capacidad con trastorno por déficit de atención y alta capacidad con trastorno del espectro autista. Revista Educatión, v. 42, n.2, p. 645-676, 2018. Disponível em:

https://www.scielo.sa.cr/pdf/edu/v42n2/2215-2644-edu-42-02-00645.pdf. Acesso em: 21 ago. 2021.

COSTA, Danielle et al. Desenvolvimento das funções executivas, comportamento motor e habilidades socioemocionais na idade escolar. In: MIRANDA, Débora; MALLOY-DINIZ, Leandro. (orgs.). O escolar. São Paulo: Hogrefe, 2020, p. 35-48.

DARE, Lynn; NOWICKI, Elizabeth Agnes. Twice exceptionality: parent's perspectives on 2e identification. Roeper Review, v. 37, n. 4, p. 208-218, 2015. Disponível em: https://www.researchgate.net/publication/283554302 TwiceExceptionality Parents' Perspectives on 2e Identification. Acesso em: 20 ago. 2021.

FOLEY-NICPON, Megan; KIM, Ji Youn Cindy. Identifying and providing evidence-based services for twice exceptional students. In: Pfeiffer, Steven (Ed.). Handbook of giftedness in children: psychoeducational theory, research and best practices. Singapura: Springer, 2018. p. 349-362.

FRANCE, Christopher et al. Application of the competency model to clinical health psychology. Professional Psychology: Research and Practice, v. 39, p. 573-580, 2008. Disponível em: https://psycnet.apa.org/doi/10.1037/0735-7028.39.6.573. Acesso em: 20 ago. 2021.

GENTRY, Marcia; FUGATE, Matthew. Attention-deficit/hyperactivity disorder in gifted students. In: PFEIFFER, Steven (Ed.). APA Handbook of giftedness and talent. Washington: American Psychological Association, 2018. p. 575-584.

GILMAN, Barbara Jackson et al. Critical issues in the identification of gifted students with co-existing disabilities: the twice-exceptional. SAGE Open, v. 3, p. 1-16, 2013. Disponível em: https://journals.sagepub.com/doi/10.1177/2158244013505855. Acesso em: 20 ago. 2021.

GUIMARÃES, Tânia Gonzaga; ALENCAR, Eunice Maria Lima Soriano. Dupla excepcionalidade, superdotação e transtorno de Asperger: contribuições teóricas. Revista AMAzônica, v. 10, n. 3, p. 95-108, 2012. Disponível em: https://dialnet.unirioja.es/servlet/articulo? codigo=4047470. Acesso em: 20 ago. 2021.

JOSEPHSON, Janet; WOLFGANG, Charlton; MEHRENBERG, Rich. Strategies for supporting students who are twice-exceptional. The Journal of Special Education Apprenticeship, v. 7, n. 2, article 8, 2018. Disponível em: https://files.eric.ed.gov/fulltext/EJ1185416.pdf. Acesso em: 20 ago. 2021.

KAUFMANN, Felice; KALBFLEISCH M. Layne, CASTELLANOS, F. Xavier. Attention deficit disorders and gifted students: what do we really know?, Storrs: National Research Center on the Gifted and Talented, University of Connecticut; 2000. Disponível em: https://nrcgt.uconn.edu/newsletters/fall004. Acesso em: 20 ago. 2021.

LANG, Margherita et al. Cognitive Profile of Intellectually Gifted Adults: Analyzing the Wechsler Adult Intelligence Scale. Assessment, v. 26, n. 5., 929-943, 2019. Disponível em:

https://pubmed.ncbi.nlm.nih.gov/28948838. Acesso em: 20 ago. 2021. 
LEE, Kelly; OLENCHAK, Richard. Accepting exceptionality: giftedness and ADHD. In: WALLACE, Belle; SISK, Dorothy; SENIOR, John (Eds.). The SAGE Handbook of gifted and talented education. 55 City Road: SAGE Publications, 2018. p. 129-143.

LUPART, Judy; TOY, Royal. Twice exceptional: multiple pathways to success. In: SHAVININA, Lavina (Ed.). International Handbook on Giftedness. Springer, 2009. p. 507-525.

MAGNIN, Eloi; MAURS, C. Attention-deficit/hyperactivity disorder during adulthood. Revue Neurologique, v.173, n.7-8, p. 506-515, 2017. Disponível em: https://pubmed.ncbi.nlm.nih.gov/28844700. Acesso em: 20 ago. 2021.

MATTA, Michael; GRITTI, Emanuela Saveria; LANG, Margherita. Personality assessment of intellectually gifted adults: A dimensional trait approach. Personality and Individual Differences, v. 140, p. 2126, 2019. Disponível em: https://psycnet.apa.org/record/2019-14660-004. Acesso em: 20 ago. 2021. MAYES, Renae; MOORE, James. The intersection of race, disability and giftedness: understanding the education needs of twice-exceptional, Africa American students. Gifted Child Today, v. 39, n. 2, p. 98 104, 2016. Disponível em: https://journals.sagepub.com/doi/10.1177/1076217516628570. Acesso em: 20 ago. 2021.

MCCOACH, Betsy; SIEGLE, Del; RUBENSTEIN, Lisa DaVia. Pay Attention to Inattention: Exploring ADHD Symptoms in a Sample of Underachieving Gifted Students. Gifted Child Quarterly, v. 64, n. 2, p. 100-116, 2020. Disponível em: https://journals.sagepub.com/doi/abs/10.1177/0016986219901320?journalCode=gcqb. Acesso em: 20 ago. 2021.

MECCA, Tatiana; SÁ, Cristina.; MACEDO, Elizeu. Avaliação de memória e inteligência em préescolares. In: DIAS, Natalia; SEABRA, Alessandra (orgs.). Neuropsicologia com pré-escolares: avaliação e intervenção. São Paulo: Pearson Clinical Brasil, 2018, p. 81-100.

METTRAU, Marsyl Bulkool; REIS, Hyadéa Maria Marino de Sant'Anna. Políticas públicas: altas habilidades/superdotação e a literatura especializada no contexto da educação especial/inclusiva. Ensaio: Avaliação em Políticas Públicas, Rio de Janeiro, v. 15, n. 57, p. 489-510, 2007. Disponível em: https://www.scielo.br/j/ensaio/a/46VNwfzHwsZdsn5dQC3WJTp/abstract/?lang=pt. Acesso em: 20 ago. 2021.

MICHELS, Maikon de Sousa; GONÇALVES, Hosana Alves. Funções executivas em um caso de TDAH adulto: a avaliação neuropsicológica auxiliando o diagnóstico e o tratamento. Revista de Neuropsicologia Latinoamericana, v. 6, n.2, p. 35-41, 2014. Disponível em: http:/ / pepsic.bvsalud.org/scielo.php?script=sci arttext\&pid=S2075-94792014000200005. Acesso em: 20 ago. 2021.

MOSQUERA, Juan José Mourino; STOBAUS, Claus Dieter; FREITAS, Soraia Napoleão. Altas habilidades/superdotação no transcurso da vida: da infância à adultez. In: VIRGOLIM, Angela; KONKIEWITZ, Elisabete Castelon (orgs.). Altas habilidades/superdotação, inteligência e criatividade: uma visão multidisciplinar. Campinas: Papirus, 2014. p. 265-282.

MULLET, Dianna; RINN, Anne. Giftedness and ADHD: Identification, Misdiagnosis, and Dual Diagnosis. Roeper Review, v.37, n.4, p. 195-207, 2015. Disponível em: https://www.tandfonline.com/doi/abs/10.1080/02783193.2015.1077910. Acesso em: 20 ago. 2021.

NAKANO, Tatiana de Cassia; SIQUEIRA, Luciana Gurgel Guida. Revisão de publicações periódicas brasileiras sobre superdotação. Revista de Educação Especial, Santa Maria, v. 25, n. 43, p. 249-66, 2012. Disponível em: https://periodicos.ufsm.br/educacaoespecial/article/view/3615. Acesso em: 20 ago. 2021. 
OGEDA, Clarissa Maria de Marques; CHACON, Miguel Claudio Moriel. Dupla excepcionalidade e superdotação no TDAH. Diálogos e Perspectivas em Educação Especial, v.7, n.1, p. 101-116, 2020. Disponível em:

https://revistas.marilia.unesp.br/index.php/dialogoseperspectivas/article/view/9825. Acesso em: 20 ago. 2021.

OUROFINO, Vanessa T. A. T. de; FLEITH, Denise de Souza. Um estudo comparativo sobre a dupla personalidade superdotação/hiperatividade. Avaliação Psicológica, Porto Alegre, v. 4, n. 2, p.165-182, 2005. Disponível em: http://pepsic.bvsalud.org/scielo.php?script=sci arttext\&pid=S167704712005000200008. Acesso em: 20 ago. 2021.

READY, Rebecca; VEAGUE, Heather Barnett. Training in psychological assessment: Current practices of clinical psychology programs. Professional Psychology: Research and Practice, v. 45, n. 4, p. 278 282, 2014. Disponível em: https://psycnet.apa.org/record/2014-32671-008. Acesso em: 20 ago. 2021.

REY, André. Teste de Cópia e Reprodução de Memória de Figuras Complexas de Rey. Manual. Trad. M. Oliveira e S. Rigoni. São Paulo: Casa do Psicólogo, 2014.

REY, André. Teste de Aprendizagem Auditivo-Verbal-RAVLT. Manual. Trad. Leandro Malloy-Diniz e J. Paula. São Paulo: Vetor, 2018.

REY-CASSERLY, Celiane; ROPER, Brad; BAUER, Russell. Application of a competency model to clinical neuropsychology. Professional Psychology: Research and Practice, v. 43, n. 5, p. 422-431,

2012. Disponível em: https://psycnet.apa.org/record/2012-27680-003. Acesso em: 20 ago. 2021.

RODRIGUES, Jaqueline Carvalho; MINÁ, Camila Schorr.; SALLES, Jerusa Fumagalli de . Anele 3 TLPP - Tarefa de Leitura de Palavras e Psendopalavras. São Paulo: Vetor, 2017a.

RODRIGUES, Jaqueline Carvalho; MINÁ, Camila Schorr; SALLES, Jerusa Fumagalli de. Anele 3 TEPP - Tarefa de Escrita de Palavras e Pseudopalavras. São Paulo: Vetor, 2017b.

RUEDA, Fabián. Bateria Psicológica para Avaliação da Atenção - BPA. São Paulo: Vetor, 2013.

SILVERMAN, Linda Kreger. Hidden treasures: twice exceptional students. In:

WALLACE, Belle; SISK, Dorothy; SENIOR, John (Eds.). The SAGE Handbook of gifted and talented education. 55 City Road: SAGE Publications, 2018. p. 144-158.

STAKE, Robert. Qualitative Case Studies. In: DENZIN, Norman; LINCOLN, Yvonnas (Eds.). Handbook of qualitative research. Newsbury Park: Sage, 2006. p. 443-466.

STRICKER, Johannes; BUECKER, Susanne; SCHNEIDER, Michael; PRECKEL, Franzies. Intellectu al Giftedness and Multidimensional Perfectionism: a Meta-Analytic Review. Educational Psychology Review, v. 32, p. 391-414, 2020. Disponível em: https://link.springer.com/article/10.1007/s10648-019-09504-. Acesso em: 20 ago. 2021.

SZYMANSKI, Antonia; WRENN, Melissa. Growing Up With Intensity: Reflections on the Lived Experiences of Intense, Gifted Adults. Roeper Review, v.41, n. 4, p. 243-257, 2019. Disponível em: https://www.tandfonline.com/doi/abs/10.1080/02783193.2019.1661054. Acesso em: 20 ago. 2021.

TAYLOR, Rebecca; OBERLE, Eva; DURLAK, Joseph; WEISSBERG, Roger. Promoting positive youth development through school-based social and emotional learning interventions: A meta-analysis of follow-up effects. Child Development, v. 88, n. 4, p. 1156-1171, 2017. Disponível em: https://pubmed.ncbi.nlm.nih.gov/28685826. Acesso em: 20 ago. 2021.

TENTES, Vanessa Terezinha Alves; FLEITH, Denise de Souza; ALMEIDA, Leandro. Novos paradigmas para a educação dos superdotados: a questão dos estudantes underachievers e com dupla 
excepcionalidade. Portugal: Editora Alínea, 2016, p. 137-155. Disponível em:

http://hdl.handle.net/1822/50154. Acesso em 13. Set. 2021.

VIAPIANA, Vanisa Fante et al. Evidências de Validade do Subteste Aritmética do TDE-II: da psicometria moderna à neuropsicologia cognitiva. Neuropsicologia Latinoamericana, v. 8, n. 2, p. 16-26, 2016. Disponível em:

https://www.neuropsicolatina.org/index.php/Neuropsicologia Latinoamericana/article/view/306. Acesso em: 15 Set 2021.

VILARINHO-REZENDE, Daniela; FLEITH, Denise de Souza; ALENCAR, Eunice Maria Lima Soriano. Desafios no diagnóstico de dupla excepcionalidade: um estudo de caso. Revista de Psicología, v. 34, n. 1, p. 61-84, 2016. Disponível em:

http://www.scielo.org.pe/scielo.php? script=sci arttext\&pid=S0254-92472016000100004. Acesso em: 20 ago. 2021.

VO'T'TER, Bernadette; SCHNELL, Tatjana. Bringing. Giftedness to Bear: Generativity, Meaningfulness, and Self-Control as Resources. Frontiers in Psychology, v. 10, 1972, 2019. Disponível em: https://www.frontiersin.org/articles/10.3389/fpsyg.2019.01972/full. Acesso em: 20 ago. 2021.

WECHSLER, David. Escala de Inteligência Wechslerpara Adultos: WAIS-III. Manual para Administraşão e Avaliação. Trad. Elizabeth Nascimento. 12. ed. São Paulo: Casa do Psicólogo, 2004.

WIRTHWEIN, Linda; ROST, Detlef. Giftedness and subjective well-being: A study with adults. Learning and Individual Differences, v. 21, 182-186, 2011. Disponível em:

https://www.sciencedirect.com/science/article/abs/pii/S1041608011000069. Acesso em: 20 ago. 2021.

WORMALD, Catherine; BANNISTER-TYRRELL, Michelle. Teacher's knowledge and understandings of twice exceptionality across Australia. In: SMITH, Susen (Ed.). Handbook of giftedness and talent development in the Asia-Pacific. Singapura: Springer, 2019. p. 1-26. Disponível em: https://www.researchgate.net/publication/344896145 Twice-

Exceptionality in Australia Prevalence Estimates. Acesso em: 20 ago. 2021.

ZAIA, Priscila; NAKANO, Tatiana de Cassia. Escala de identificação das altas habilidades/superdotação: evidências de validade de critério. Revista Iberoamericana de Diagnóstico y Evaluación e Avaliação Psicológica, v. 55, n. 2, p. 31-41, 2020. Disponível em: https://www.aidep.org/sites/default/files/2020-04/RIDEP55-Art3.pdf. Acesso em: 20 ago. 2021.

ZENASNI, Franck. et al. How does creative giftedness differ from academic giftedness? A multidimensional conception. Learning and Individual Differences, v. 52, p. 216-223, 2016. Disponível em: https://www.sciencedirect.com/science/article/abs/pii/S1041608016302047. Acesso em: 20 ago. 2021. 